\title{
Pump-Probe Studies of Carrier Capture Processes in Semiconductor Multiple-Quantum-Well Waveguides
}

\author{
Jian-Jang Huang, Ding-Wei Huang, Chung-Yen Chao, Jiun-Haw Li, \\ C. C. Yang, Ming-Ching Chen, and Hao-Hsiung Lin
}

\begin{abstract}
Carrier capture processes in multiple-quantum-well (MQW) waveguides are monitored using the time-resolved pumpprobe technique. With appropriate selections of wavelength and pulse recompression, subpicosecond pulses at various wavelengths can be obtained for both nondegenerate and degenerate pumpprobe experiments with various polarization combinations. Two samples of different quantum-well structures are compared. It is found that the carrier capture times (defined as the time period to reach probe transmission maximum after the pump) are in the range from 15 to $50 \mathrm{ps}$ in the samples used. In the degenerate pump-probe measurements, a two-component decay is observed for one of the samples in the case of TM pump and TE probe.
\end{abstract}

Index Terms - Charged carrier processor, quantum-well devices.

C ARRIER capture is referred to as the transition of an electron or hole from a quasi-continuum or unconfined state (3-D) to a confined quantum-well (QW) (2-D) state in a QW structure. On the other hand, carrier transport implies the spatial movements of carriers in the same energy state. Most carrier capture measurements actually include some carrier transport processes. In semiconductor QW lasers, the capture processes of carriers have been identified as an important factor in determining the threshold current and modulation frequency [1], [2]. From the experimental point of view, the carrier capture processes have been studied with various techniques; including luminescence spectroscopy, time-resolved photoluminescence [3], [4], and time-resolved pump-probe measurement [5], etc. The advantages of the timeresolved pump-probe technique include that it can directly monitor the time evolution of carrier dynamics. Depending on quantum well structures, carrier capture times ranging from subpicosecond to several tens of picoseconds have been reported [6]-[8]. Besides the inconsistent experiment results, theoretical predictions added to the controversy of this issue [9], [10]. It is usually believed that the capture of hole is faster than that of electron. In a time-resolved pump-probe measurement, we can set both pump and probe wavelengths corresponding to the transition between the unconfined states of electron and hole (degenerate pump-probe) or set the pump wavelength as above but set the probe wavelength

Manuscript received November 27, 1996; revised January 24, 1997. This work was supported by National Science Council, Republic of China, under Grant NSC 85-2215-E-002-003 and Grant NSC 85-2215-E-002-004.

The authors are with the Institute of Electro-Optical Engineering and Department of Electrical Engineering, National Taiwan University, Taipei, Taiwan, R.O.C

Publisher Item Identifier S 1041-1135(97)03227-8 corresponding to the confined states of both kinds of carriers (nondegenerate pump-probe). The degenerate pump-probe measurement monitors the relaxation process of the kind of carrier of fast capture. The nondegenerate pump-probe measurement monitors the relaxation processes of both kinds of carriers. In other words, the degenerate measurement can provide the information about the required time period for the carrier of fast capture to leave the unconfined state and the nondegenerate measurement provides that of the required time period for both carriers to arrive at the respective confined states. In this letter, we report the results of degenerate and nondegenerate pump-probe measurements of carrier capture processes in semiconductor multiple-quantum-well (MQW) waveguides. We compare the results of two different quantum well structures.

Both samples had intrinsic $\mathrm{GaAs}_{\mathrm{A}}-\mathrm{Al}_{0.18} \mathrm{Ga}_{0.82} \mathrm{As} \mathrm{MQW}$ structures with ridge-loading waveguide configurations. Sample A consisted of 55 quantum wells with well (barrier) thickness $7 \mathrm{~nm}(12 \mathrm{~nm})$. Sample B consisted of 110 quantum wells with well (barrier) thickness $3.5 \mathrm{~nm}(5.5 \mathrm{~nm}$ ) (a superlattice). Hence, the waveguides of both samples had thicknesses of about $1 \mu \mathrm{m}$. According to the results of photoreflectance measurements, confirmed by theoretical calculations, at room temperature the band edge of the barrier-barrier transition corresponded to about $750 \mathrm{~nm}$ in wavelength in both samples; that of the C1-HH1 transition in sample A (B) corresponded to $841 \mathrm{~nm}(815 \mathrm{~nm})$ in wavelength; and that of the C1-LH1 transition in sample A (B) corresponded to $831 \mathrm{~nm}(806 \mathrm{~nm})$ in wavelength. Those wavelengths with various polarization combinations were used to conduct the pump-probe experiments. To separate the pump and probe beams easily; the pump and probe pulses were always in the perpendicular polarizations.

Fig. 1 shows the experimental setup 80 -fs transform-limited pulses, centered at $790 \mathrm{~nm}$, from a Ti:sapphire laser were first end-coupled into a 14-cm-long ordinary communication fiber. With the self-phase modulation effect in the fiber, the spectrum of the pulses was broadened to about $100 \mathrm{~nm}$ in full-width at half-maximum (FWHM). Meanwhile, the pulses became chirped and broadened. A grating-slit set was then used in either pump or probe arm to first select the required central wavelength and spectral width (about $12 \mathrm{~nm}$ ), and then recompress the pulses down to about $150 \mathrm{fs}$. After the nonpolarization beam splitter, the recombined pump and probe beams were end-coupled into the waveguide samples which were maintained at $23^{\circ} \mathrm{C}$. After the samples, the pump pulses were removed by a polarization beam splitter with a 


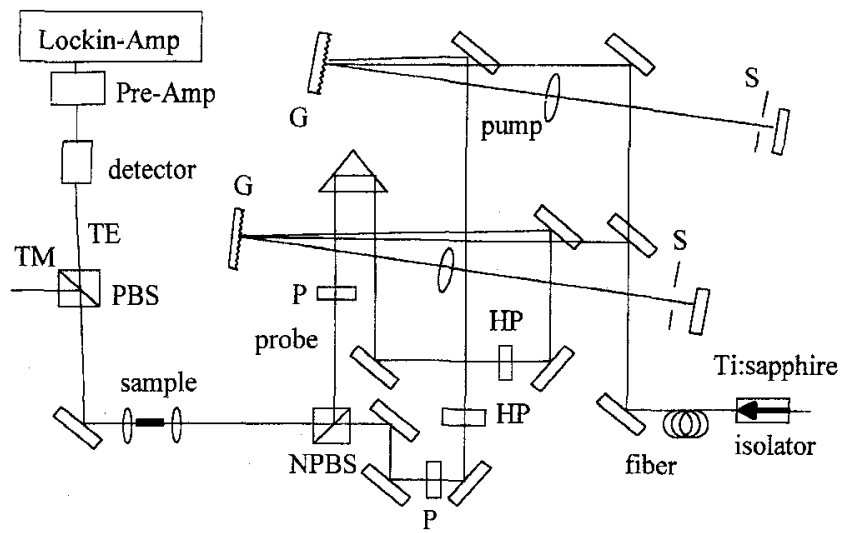

Fig. 1. Experimental setup. G: grating. HP: halfwave-plate. NPBS: nonpolarization beam splitter. P: polarizer. PBS: polarization beam splitter. S: slit.

high extinction ratio $\left(10^{5}\right)$ and the transmitted probe pulses were recorded. The operation of the experiment was computer controlled. We measured the changes of probe transmission due to the affects of the pump pulses as a function of the delay time between the pump and probe pulses. In the degenerate experiments, the pump pulses (which had a pulse energy about ten times that of the probe pulses) generated carriers in the barrier states and the probe pulses experienced the reduced absorption at the same wavelength. Because the carriers would relax into the well subbands, we expected the decrease of probe transmission during the carrier capture processes. In these experiments, the computer recorded the transmitted probe power, normalized by the maximum transmission. In the nondegenerate experiments, the probe pulses monitored the reduced absorption at the wavelengths correponding to the subband transitions. We expected the increasing trend of pulse transmission after the carriers were generated. In these experiments, the computer recorded the normalized differential transmission of probe, i.e., $\left(T-T_{0}\right) / T_{0}$, where $T$ and $T_{0}$ are the transmitted probe powers with and without the pump pulse, respectively. The average power of the pump pulses was kept at $1.5 \mathrm{~mW}$. It was estimated that a $50 \%$ coupling efficiency was achieved in end-coupling the laser into the waveguide samples.

The normalized differential transmission of the probe in the nondegenerate experiment with the pump in the TM polarization $(740 \mathrm{~nm})$ and probe in the TE polarization $(841$ $\mathrm{nm}$ for sample $\mathrm{A}$ and $815 \mathrm{~nm}$ for the sample B, corresponding to the transition between $\mathrm{Cl}$ and HH1) is shown in Fig. 2 . Similar results with the pump in the TE polarization and probe in the TM polarization ( $831 \mathrm{~nm}$ for sample A and 806 $\mathrm{nm}$ for sample $\mathrm{B}$, corresponding to the transition between $\mathrm{C} 1$ and LH1) is shown in Fig. 3. In Figs. 2, 3, and the following data figures, the solid and dashed curves are used to represent the data of samples A and B, respectively. In Fig. 2, the differential transmission of sample A reaches its maximum at about $25 \mathrm{ps}$ after the excitation of the pump pulse, while in sample B it reaches its maximum at about 18 ps after the excitation. After the transmission maximum, the differential transmission is expected to decrease because there is more carrier loss through recombination and other relaxation

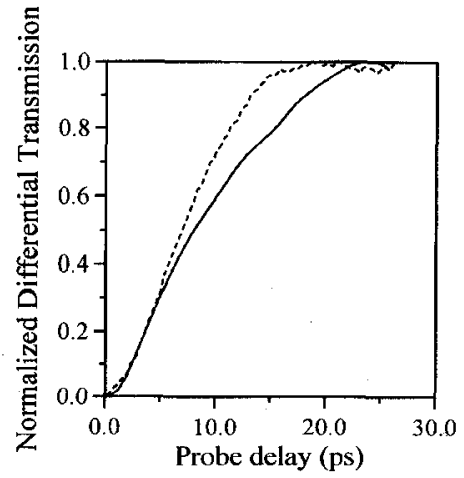

Fig. 2. Normalized differential transmission in the nondegenerate experiments with samples A (solid curve) and B (dashed curve) (TM pump and TE probe).

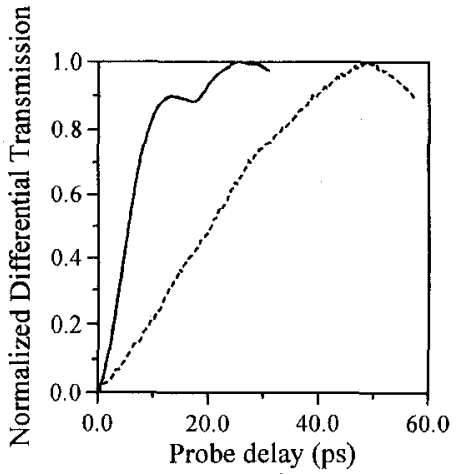

Fig. 3. Normalized differential transmission in the nondegenerate experiments with samples A (solid curve) and B (dashed curve) (TE pump and TM probe).

processes than carrier gain through the capture processes. With the probe pulse wavelength associated with light hole subbands, the carrier capture times are different, as shown in Fig. 3. Here, the transmission maximum of sample $A$ is reached about 25 ps after the excitation, while that of sample B is about $47 \mathrm{ps}$ after the excitation. The slower capture process in sample B reflects the possibility that while the hole density in the light hole subband increases through the capture of holes from the barrier states, it decreases through the relaxation into the heavy hole subband besides electron-hole recombination. Generally speaking, our measured values are larger than those reported [5]. The differences can be attributed to the fact that in the samples we used, the QW numbers are much more than those reported. The drift and tunneling of carriers among wells can be two of the reasons for the larger capture times [8]. Since the two sample structures are quite different, the comparisons of the results between the two samples provide little information about the well width dependence of the capture time [5].

The normalized transmission of the probe in the degenerate experiment with the pump in the TM polarization $(740 \mathrm{~nm})$ and probe in the TE polarization (also $740 \mathrm{~nm}$ ) is shown in Fig. 4. Similar results with the pump in the TE polarization and probe in the TM polarization are shown in Fig. 5. In such an experiment, after the excitation of the pump pulse, the transmission of the probe pulse increases rapidly and then decreases with the relaxation of carriers into the quantum 


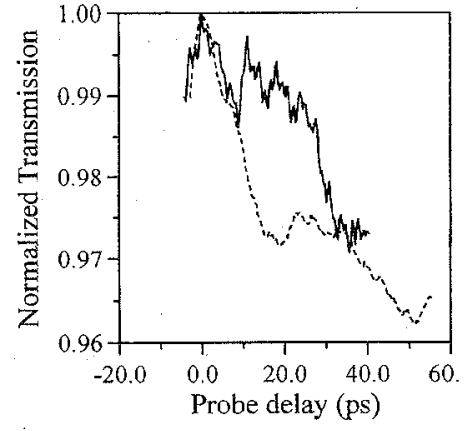

Fig. 4. Normalized transmission in the degenerate experiments with samples A (solid curve) and B (dashed curve) (TM pump and TE probe).

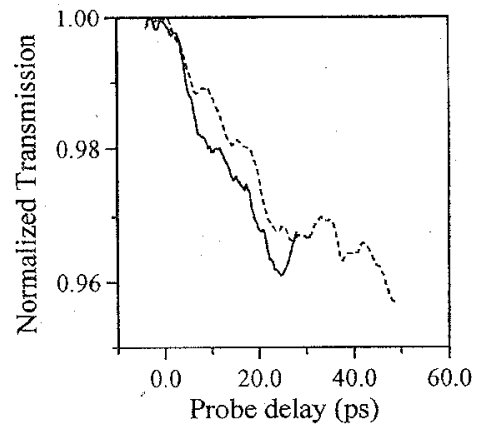

Fig. 5. Normalized transmission in the degenerate experiments with samples A (solid curve) and B (dashed curve) (TE pump and TM probe).

well states. In sample A, after the rapid increase of the probe transmission, there exists a two-component decay in the case of Fig. 4. This decay trend was not observed in sample B. It was not observed in either sample in the case of Fig. 5. From Fig. 5, we can see that the decay rates of the two samples are about the same.

The comparison between Figs. 2 and 3 shows the effects of different polarizations. The reverse relative position of the solid and dashed curves is the most prominent difference in these two figures. In Fig. 2, the faster increase of probe transmission in sample B may have a complicated cause. The most intuitive reason is that the carrier released energies are different between the two samples. The sample with appropriate LO phonon relaxation would lead to a shorter capture time. The major difference in the case of TM probe from the case of TE probe is that the LH1 involved in the transition is not the ground subband for holes. Hence, not all the holes excited in the barrier states can reach the band edge of the LH1 subband. Some holes may relax directly into the HH1 subband. Also, the holes relax into the band edge of the LH1 subband may further relax down to the HH1 subband before they are used for either stimulated or spontaneous transition. This hole leakage from the LH1 subband may be significant in sample B. This faster leakage can be one of the reasons for the slower increase of probe transmission in sample B, as shown in Fig. 3 .

The most prominent feature shown in Figs. 4 and 5 is the two-component decay in sample $A$ in the case of TM pump and TE probe. One possible reason for this phenomenon is that the band structure of this sample is formed in such a way that there exist states for electrons and/or holes into which the carriers can at least partially relax and satisfy the conditions of energy and momentum conservation for the TE probe transition. Therefore, even carriers (at least one kind) leave the states corresponding to the TM pump transition, the TE probe (not the TM probe) can still monitor the carriers in the case that the probe has the same photon energy as the pump. The two-component (slower) decay in Fig. 4 is consistent with the slower increase of probe transmission in Fig. 2 for sample A.

In summary, the carrier capture processes of two different QW waveguides were observed using the pump-probe technique. As for the future work, carrier density dependence of capture time, which has been somewhat studied [11], is an issue to be measured in our next-step experiment. Meanwhile, a physical model is to be built for explaining the observed phenomena.

\section{REFERENCES}

[1] P. W. M. Blom, J. E. M. Haverkort and J. H. Wolter, "Optimization of barrier thickness for efficient carrier capture in graded-index and separate-confinement multiple quantum well lasers," Appl. Phys. Lett., vol. 56, pp. 2767-2769, 1991.

[2] N. Tessler, R. Nagar and G. Eisenstein, "Structure dependent modulation responses in quantum-well lasers," IEEE J. Quantum Electron., vol. 28, pp. 2242-2250, 1992.

[3] B. Deveaud, J. Shah, T. C. Damen and W. T. Tsang, "Capture of electrons and holes in quantum wells," Appl: Phys. Lett., vol. 53, pp. $1886-1888,1988$.

[4] R. Kersting, R. Schwedler, K. Wolter, K. Leo and H. Kurz, "Dynamics of canrier transport and carrier capture in $\mathrm{In}_{1-x} \mathrm{Ga}_{x} \mathrm{As} / \mathrm{InP}$ heterostructures," Phys. Rev. B, vol. 46, pp. 1639-1648, 1992.

[5] P. W. M. Blom, C. Smit, J. E. M. Haverkort and J. H. Wolter, "Carrier capture into a semiconductor quantum well," Phys. Rev. B, vol. 47, pp. 2072-2081, 1993.

[6] J. Feldmann, G. Peter, E. O. Gobel, K. Leo, H. J. Polland, K. Ploog, K. Fijiwara and T. Nakayama, "Carrier trapping in single quantum wells with different confinement structures," Appl. Phys. Lett., vol. 51, pp. 226-228, 1987.

[7] B. Devaud, F. Clerot, A. Regreny, K. Fujiwara, K. Mitsunage and J. Ohta, "Capture of photoexcited carriers by a laser structure," Appl. Phys. Lett., vol. 54, pp. 2646-2648, 1989.

[8] S. Morin, B. Deveaud, F. Clerot, K. Fujiwara and K. Mitsunaga, "Capture of photoexcited carriers in a single quantum well with different confinement structures," IEEE J. Quantum Electron., vol. 27, pp. $1669-1675,1991$.

[9] J. A. Brum, T. Weil, J. Nagle and B. Vinter, "Calculation of carrier capture time of a quantum well in graded-index separate-confinement heterostructures," Phys. Rev. B, vol. 34, pp. 2381-2384, 1986.

[10] C. Y. Tsai, C. Y. Tsai, Y. H. Lo and L. F. Eastman, "Carrier DC and AC capture and escape times in quantum-well lasers," IEEE Photon. Technol. Lett., vol. 7, pp. 599-601, 1995.

[11] D. Y. Oberli, J. Shah, J. L. Jewell, T. C. Damen and N. Chand, "Dynamics of carrier capture in an InGaAs/GaAs quantum well trap," Appl. Phys. Lett., vol. 54, p: 1028, 1989. 